\title{
A Vibration-Based Approach for the Estimation of the Loss of Composite Action in Timber Composite Systems
}

\author{
Ulrike Dackermann ${ }^{1, a}$, Jianchun $\mathrm{Li}^{1, \mathrm{~b}}$, Rajendra Rijal ${ }^{1, \mathrm{c}}$ and Bijan Samali ${ }^{1, \mathrm{~d}}$ \\ ${ }^{1}$ University of Technology, Sydney, Centre for Built Infrastructure Research, Faculty of Engineering \\ and Information Technology, P.O. Box 123, Broadway, NSW 2007, Australia \\ aUlrike.Dackermann@uts.edu.au, bJianchun.Li@uts.edu.au, 'Rajendra.Rijal@student.uts.edu.au, \\ and 'Bijan.Samali@uts.edu.au
}

Keywords: Composite action, composite flooring system, Damage Index method, damage detection, timber structures, structural vibration.

\begin{abstract}
This paper presents a novel approach for the determination of the loss of composite action for timber composite systems using only measurements from non-destructive vibration testing. Traditionally, the composite action of a system is evaluated from static load testing using deflection measurements. However, static load testing is expensive, time consuming and inappropriate for existing flooring systems. The method proposed in this paper is based on the Damage Index (DI) method, which uses changes in modal strain energies, to detect locations and severities of damage. In the proposed method, a new Loss of Composite Action Index (LCAI), which is derived from direct mode shape measurements obtained from dynamic testing, is introduced to evaluate the loss of composite action. The proposed method is tested and validated on numerical and experimental models of a timber composite beam structure, which consists of two timber components that are connected with different numbers of screws to simulate various degrees of partial composite states. The results obtained from the new method are very encouraging and show a clear trend of the proposed dynamic-based LCAI in indicating the loss of composite action in the investigated timber composite structure.
\end{abstract}

\section{Introduction}

Timber composite systems, such as timber-concrete composite (TCC) flooring systems, are new construction techniques to resolve vibration susceptibility and low impact insulation issues concerned with light-weight timber floors. Concrete performs well in compression while timber is stronger in tension, therefore, the tensile resistance of the timber joist can be used in a similar fashion as steel reinforcement in conventional concrete construction. In the design of TCC flooring systems, the concrete topping is in compression and the area in tension is replaced with timber to utilize the strength and stiffness of both materials for greatest advantage. To transfer shear forces from the concrete slab to the timber joists, shear connectors are essential to provide composite action in the cross-section. The integrity of such connection system is crucial for the accurate functioning and efficiency of the timber composite systems as the advantages of the system cannot be achieved unless a strong connection can be assured. Hence, in order to ensure a composite system is performing satisfactorily under both, static and dynamic actions, it is essential to assess the condition of the connection system at different stages during the life span of the system.

Traditionally, the composite action of a composite system is determined from static load testing using deflection measurements. This testing method is based on the fact that the effective bending stiffness of a composite system is highly dependent on the shear bond coefficient of the interface, $\gamma$. A system with $\gamma=0$ means the layers are acting totally independently and there is no shear transfer between the layers. Such system is known as 'fully non-composite'. This results in a large amount of slip between the layers and large deflection. A flooring system with $\gamma=1$ represents a homogenous composite action with no slip in the interface and the smallest deflection possible and is termed 'fully composite' [1]. The actual behaviour of a real structural composite system is 'partially composite', i.e. it exhibits a deflection that lies between these two extremes. The degree of composite action is a 
measure of how close any interconnection comes to achieving the fully composite extreme. Pault [2] quantified the degree of composite action (composite efficiency) based on static test results using the following equation:

$$
C A(\%)=\frac{D_{N}-D_{I}}{D_{N}-D_{C}} \times 100,
$$

where $C A(\%)$ is the composite action in $\%, D_{N}$ is the theoretical fully non-composite deflection, $D_{I}$ is the measured deflection for partial composite action, and $D_{C}$ is the theoretical full composite deflection of the system [3]. The resulting static-based Loss of Composite Action (LCA) is therefore defined as:

$$
L C A(\%)=100 \%-\frac{D_{N}-D_{I}}{D_{N}-D_{C}} \times 100 .
$$

While the assessment of a composite system using static load testing is feasible; for existing flooring systems, such testing is expensive, time consuming and cumbersome to carry out. In addition, already compromised connection systems can get damaged further by conducting such load testing. In order to provide an alternative to load testing, the research presented in this paper explores the possibility of using changes in the dynamic characteristics of a composite system to assess the condition of its connection system. The basis of such approach is that damage in a structure, such as the loosening of shear connections, changes not only the physical properties of the structure (such as stiffness, mass and boundary conditions) but also its dynamic characteristics (e.g. natural frequencies, damping ratios and mode shapes). Therefore, by analysing a structure's dynamic properties from structural vibration, any damage, including the failure of shear connectors, can be identified. The research field of vibration-based damage detection is not new, and over the past three decades much work has been undertaken and many algorithms have been developed that are concerned with the delamination and damage identification of structural systems. Comprehensive literature reviews on vibration-based damage detection methods can be found in [4-6]. However, only very limited work has been conducted in the area of using dynamic approaches for the composite action determination in timber composite systems and no vibration-based method is currently available that can give satisfactory assessment on the condition of the connection system of a timber composite structure.

The research in this paper presents an investigation on using vibration measurements for the evaluation of the connection integrity of timber composite systems. The proposed method is based on the Damage Index (DI) method that was initially developed by Stubbs, Kim \& Topole [7], and which was subsequently successfully applied by many researchers [8-12]. In this method, relative differences in modal strain energy (derived from mode shape measurements) between an undamaged and damaged structure are utilised to locate and quantify damage. In the presented research, the method is modified and extended to enable the evaluation of the degrees of composite action in a timber composite system. To verify the proposed method, it is applied to numerical and experimental models of a timber composite beam structure, where the number of the shear connection elements are changed, and the different loss of composite action scenarios are assessed using the new method.

\section{Proposed Methodology}

The proposed approach for the vibration-based composite action identification of the connection system in a composite structure involves the following procedure. First, the composite structure is dynamically tested in order to determine the modal parameters of the structure including natural frequencies and mode shapes. Second, following the algorithm of the DI method, the damage severity index $\alpha_{i j}$ (see detailed derivations below (Eq. 3 to Eq. 6)) is determined from mode shape measurements of different modes. Third, the proposed dynamic-based Loss of Composite Action 
Index (LCAI) (see Eq. 7 below) is calculated considering all $\alpha_{i j}$ values along the length of the beam and all modes. To validate the method, a laboratory model of a timber composite beam is built and various configurations of the shear connection system are dynamically tested. In addition, a corresponding numerical model is created and by solving the eigenvalue problem, the dynamic characteristics are determined. By applying the proposed method to both, the experimentally and numerically derived mode shapes, the new method is evaluated. The following section gives a brief overview on the DI method including the associated equations as well as the equation of the proposed dynamic-based LCAI.

The Damage Index Method. The DI method utilises an indicator based on modal strain energies, which are a function of relative changes in mode shape curvatures of a structure before and after damage, to detect, locate and quantify damage. The strain energy in a Bernoulli-Euler beam associated with a particular mode shape $\phi_{i}$ can be calculated from Eq. 3. By subdividing the Euler-Bernoulli beam and associating the modal strain energy to an element $j$ and relating the damaged $^{*}$ to the undamaged state, the so-called damage index $\beta_{i j}$ is obtained from Eq. 4 . To establish a comparative basis for different modes, the damage index $\beta_{i j}$ is transformed into the standard normal space, and the normalised damage index $Z_{i j}$ is derived, which is an indicator for the location of damage. The estimation of the damage severity for an element $j$ can be formulated by Eq. 5, with $\alpha_{i j}$ being the severity estimator. The positive $Z_{i j}$ and $\alpha_{i j}$ values of the DI method indicate the possibility of damage and can therefore be utilised to locate and quantify damage, respectively. A more detailed description of the DI method can be found in [7, 10,11].

$$
U_{i}=\frac{1}{2} \int_{0}^{L} E I\left(\phi_{i}^{\prime \prime}(x)\right)^{2} d x \text { (3) } \quad \beta_{i j}=\frac{\int_{j}\left(\phi_{i}^{\prime \prime *}(x)\right)^{2} d x \int_{0}^{L}\left(\phi_{i}^{\prime \prime}(x)\right)^{2} d x}{\int_{j}\left(\phi_{i}^{\prime \prime}(x)\right)^{2} d x \int_{0}^{L}\left(\phi_{i}^{\prime \prime *}(x)\right)^{2} d x} \text { (4) } \quad \alpha_{i j}=1-\frac{1}{\beta_{i j}}
$$

The Loss of Composite Action (LCA) Method. Based on the damage severity indicator $\alpha_{i j}$ from the DI method, the proposed dynamic-based LCAI considers all positive $\alpha_{i j}$ values from all available modes. The composite action is a global characteristic of a composite structure, and hence, the proposed LCAI combines all positive $\alpha_{i j}$ values along the length of a structure, which indicate the severity of possible damage, and all measurements of all modes. Therefore, in the proposed method, first, the mean of all positive $\alpha_{i j}$ values is independently calculated for each mode $i$. Then, the mean of the averaged positive $\alpha_{j}$ values is determined for all considered modes $M$. The vibration-based LCAI can mathematically be expressed as follows:

$$
L C A I=\frac{\left\{\sum_{i=1}^{M}\left(\sum_{j=1}^{n} \frac{\alpha_{i j}}{n}\right)\right\}}{M} \text { for } \alpha_{i j}>0 \text { and } n \leq N,
$$

where $n$ is the number of positive $\alpha_{i j}$ values, $M$ is the total number of considered modes and $N$ the total number of measurement locations. The value of $n$ can be different for different modes, but it cannot be greater than $N$.

\section{The Test Structure}

To validate the proposed dynamic-based composite action identification method, it was applied to a laboratory and a numerical model of a T-shaped timber composite beam structure. The test structure consisted of two timber elements that were connected using different numbers of connection elements (screws) to simulate various degrees of composite action. The laboratory model was manufactured and tested in the structures laboratory of the University of Technology Sydney (UTS). The numerical 
model, which was based on the experimental structure, was created using the software ANSYS.

Laboratory Timber Composite Beam. The experimental timber composite beam consisted of two hySPAN PROJECT Laminated Veneer Lumber (LVL) elements with pin-pin support conditions and a span length of $3.5 \mathrm{~m}$, as shown in Fig. 1 (a). The cross-sectional dimensions of the flange and web elements were $90 \times 45 \mathrm{~mm}$ and $90 \times 35 \mathrm{~mm}$, respectively (see Fig. 1 (b)). The two LVL web and flange elements were connected to form a T-shape with different numbers of screws. In total, five different screw connection conditions were tested, i.e. 33, 17, 9, 5 and 3 screws, with 33 screws presenting the fully composite state.

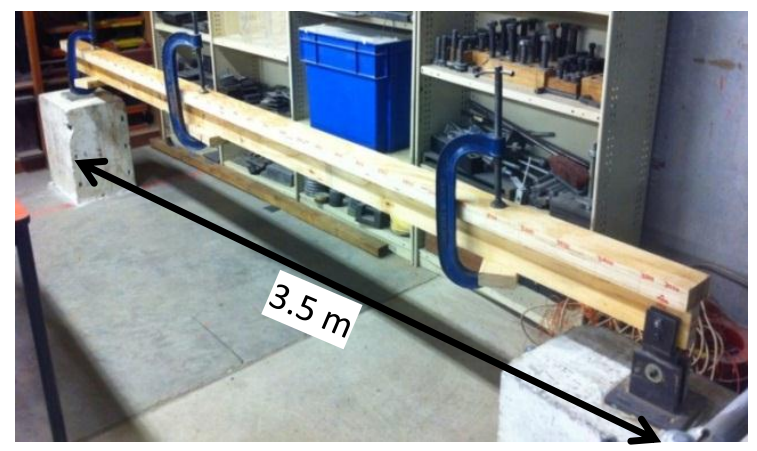

(a)

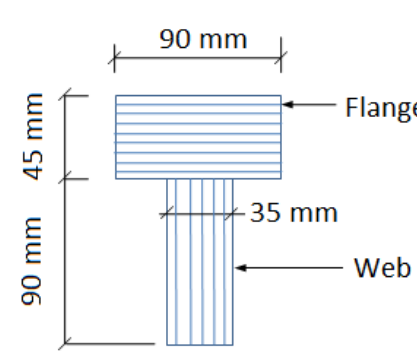

(b)

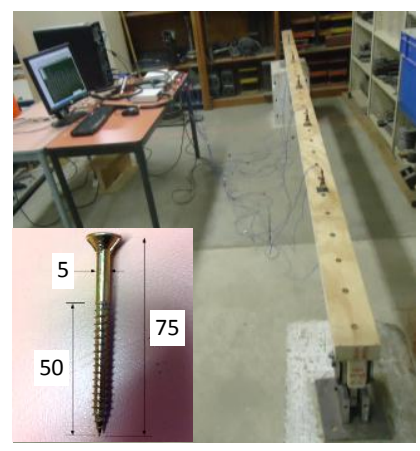

(c)

Fig. 1 Experimental timber composite beam: (a) manufacturing phase, (b) cross-sectional dimensions and (c) test set-up for MT\&EMA for beam with 33 screw connections and wood screw Type 17.

The screws used to connect the flange to the web element were normal wood screws of Type 17 (depicted in Fig. 1 (b)). The total length of a screw was $75 \mathrm{~mm}$ and the threaded length was $50 \mathrm{~mm}$. The measured shank diameter was $5 \mathrm{~mm}$. The screws were inserted from the top of the flange, with about $30 \mathrm{~mm}$ length of the screw being driven into the web element. While this paper investigates the 'loss' of composite action, for practicality reasons, the experimental testing of the composite beam structure was undertaken in reverse order, with 3 connection screws presenting the first tested composite state and 33 screws being the last tested state. As such, in the first test configuration, only three screws were used to connect the two LVL elements; two were inserted at the pin supports and one at mid span with equal spacing of $1,750 \mathrm{~mm}$. For the succeeding four composite conditions, the number of connection screws was increased to 5, 9, 17 and 33 screws. The additional screws were inserted in-between the screws of the previous configuration. Hence, the spacing between the screws in the succeeding configurations was halved from previous ones. The actual spacing between the screws for all five composite states are as follows: $110 \mathrm{~mm}$ for 33 screws, $219 \mathrm{~mm}$ for 17 screws, $438 \mathrm{~mm}$ for 9 screws, $875 \mathrm{~mm}$ for 5 screws and $1750 \mathrm{~mm}$ for 3 screws. The material properties of the LVL web and flange elements were determined by conducting a number of bending, shear, compression and tension tests in the material testing laboratories of UTS. Details of the conducted tests can be found in Zabihi et al. [13]. The determined material properties relevant for the numerical modelling of the beam are the modulus of elasticity being $13.3 \mathrm{GPa}$ and the density being $600 \mathrm{~kg} / \mathrm{m}^{3}$.

To determine the modal parameters of the laboratory timber composite beam, i.e. natural frequencies, damping ratios and mode shapes, modal testing and experimental modal analysis (MT\&EMA) was performed. The testing set-up can be seen in Fig. 1 (c). In modal testing, the beam was excited by an impact hammer and the acceleration responses were recorded by five piezoelectric accelerometers. The sensors were located on the centre line on top of the flange element with even spacing (i.e. $1 / 6^{\text {th }}, 2 / 6^{\text {th }}, 3 / 6^{\text {th }}, 4 / 6^{\text {th }}$ and $5 / 6^{\text {th }}$ of the span length). The impact location (reference or driving point) was situated at $1 / 3^{\text {rd }}$ of the span. The accelerometers used were of ICP type (model $352 \mathrm{C} 34$ ) having a sensitivity of $100 \mathrm{mV} / \mathrm{g}$ and the impact hammer was a modal hammer (model HP 086C05). A multi-channel signal conditioner (PCB model 483B03) was used to amplify and condition the time history signals from the impact hammer (input signal) and the accelerometers 
(output signal). From the signal conditioner, the input and output channels were connected to a data acquisition system through a terminal block (model SCB-68). The acquisition system had two internally synchronised acquisition cards (model NI PCI-6133) with a resolution of 14 bit and a sampling rate of $2.5 \mathrm{MS} / \mathrm{s}$, simultaneously. For all tests, the sampling rate was set to $1,000 \mathrm{~Hz}$ with 16,384 time domain data points recorded, which corresponds to a frequency bandwidth ranging from 0 to $500 \mathrm{~Hz}$ with $8,192 \mathrm{FRF}$ data points giving a frequency resolution of $0.061 \mathrm{~Hz}$ in the frequency domain. To determine FRF data in the frequency domain, the acquired time domain signals from the impact hammer and the five accelerometers were processed using the fast Fourier transform (FFT). In experimental modal analysis, the acquired signals from the impact hammer and the five accelerometers were analysed and the modal parameters of the structure were determined using the LMS CADA-X software. In the modal analysis module of LMS CADA-X [14], the frequency domain direct parameter identification (FDPI) method was used to estimate the modal parameters.

Table 1 Natural frequencies of the first five flexural modes of the experimental beam for different screw connection stages.

\begin{tabular}{|c|ccccc|}
\hline \multirow{2}{*}{$\begin{array}{c}\text { No. of } \\
\text { screws }\end{array}$} & \multicolumn{5}{|c|}{ Natural frequencies [Hz] } \\
\cline { 2 - 6 } & $f_{1}$ & $f_{2}$ & $f_{3}$ & $f_{4}$ & $f_{5}$ \\
\hline 33 & 24.1 & 85.8 & 161.4 & 256.3 & 350.6 \\
17 & 23.8 & 83.9 & 154.9 & 240.3 & 336.0 \\
9 & 23.0 & 80.4 & 139.0 & 222.1 & 318.7 \\
5 & 22.1 & 76.7 & 133.0 & 207.6 & 269.1 \\
3 & 19.3 & 57.7 & 118.4 & 156.9 & 213.4 \\
\hline
\end{tabular}

In total, the first five flexural modes could be determined including the natural frequencies, damping ratios and mode shapes. While the first three modes could be identified with high reliability, the determination of the higher modes (modes 4 and 5) was difficult due to uncertainties in the stabilisation diagram, which is the result of the limited number of measurement sensors. The determined natural frequencies of the first five modes are presented in Table 1 . The listed values are mean values of five sets of testing data resulting from five different hammer hits. From the table, it can be seen that the stiffness of the beam decreases with a decrease in the number of shear connectors, as a consequence, the natural frequencies also decrease. For the fundamental frequency, there was a large decrease of about $15 \%$ from the composite stage of 5 screws to 3 screws, while for the preceding stages of 9,17 and 33 screws, the decrements were only $4.0 \%, 3.5 \%$ and $1.3 \%$, respectively. This shows that an almost fully composite state is achieved for 33 screws and there will only be a minimal increment in natural frequency and stiffness with any further increase in the number of connection screws. More details on the experimental testing and results can be found in [15].

Numerical Timber Composite Beam. A finite element model of the experimental timber composite beam structure was created using the software ANSYS as shown in Fig. 2. All dimensions and material properties of the numerical model were identical to the values of the laboratory structure. The 2-D element PLANE42 was utilized to model both, the LVL flange and web of the composite beam structure. The boundary conditions of the beam model were set to pin-roller. Therefore, translations in global $\mathrm{X}$ and $\mathrm{Y}$ axes were restrained to simulate the pin support, while only the translation in global $\mathrm{Y}$ axis was restrained for roller support at the other end. For both supports, rotation in the global $\mathrm{Z}$ axis was allowed. It is noted that the experimental beam was supported at each end by a special type of pin support with an offset of $75 \mathrm{~mm}$ from the bottom of the web. For the numerical models, a pin-roller support system was modelled with the support connections located at the bottom of the web. This resulted in a numerical structure with good correlation in the natural frequencies and mode shapes to the experimental beam structure. 


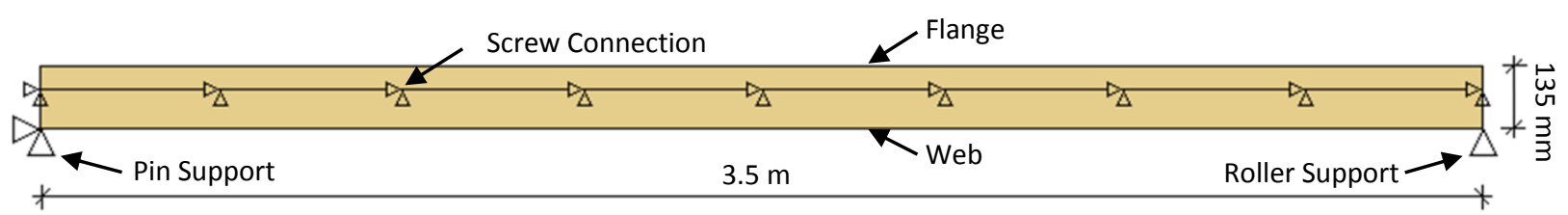

Fig. 2 2-D numerical model of timber composite beam (with 9 screw connections).

To simulate the different degrees of composite action, five numerical beam models with identical dimensions but different numbers of screw connections $(33,17,9,5$ and 3$)$ were created. Two nodes, one at the top of the web and another at the bottom of the flange, were coupled for each screw location to simulate a screw connection. The coupling of nodes constrains both, horizontal and vertical displacements. While it is more accurate to undertake push-out tests on the experimental composite structure to determine correct shear connection properties, a contact connection of two nodes was deemed accurate enough for the numerical modelling of a screw connection. To prevent the penetration of the flange and web elements during deformation, the interface between the LVL flange and web was simulated utilizing the contact and target elements CONTA171 and TARGE169. The bottom layer of the flange was defined as contact surface while the top layer of the web was defined as target surface. To identify the optimal mesh size for the numerical beam models, a sensitivity analysis was performed for both, static and modal analysis, to determine the optimum size of the elements resulting in sufficient accuracy. The optimisation parameters were the mid span deflection and first natural frequency, which were extracted from the FE models using static and modal analysis for different mesh sizes. From the sensitivity analysis it was found that an element size of not greater than $10 \times 10 \mathrm{~mm}(4,900$ elements) is appropriate for the accurate modelling of the composite beam structure. Hence, in all the FE models, the size of the elements chosen for both, flange and web components, was $5 \times 5 \mathrm{~mm}$. In total, the FE models of the beam had 18,900 elements. For each composite beam model (with different numbers of screw connections), the first five flexural modes, with their corresponding natural frequencies, damping ratios and mass normalised mode shapes, were extracted from the different numerical models by solving the eigenvalue problem utilising the modal analysis module in ANSYS. The determined natural frequencies of the first five modes of the model with 33 screws were $23.1 \mathrm{~Hz}, 88.3 \mathrm{~Hz}, 175.9 \mathrm{~Hz}, 240.3 \mathrm{~Hz}$ and $352 \mathrm{~Hz}$, respectively. A correlation analysis between the experimental and the numerical structure showed that the numerical model is fairly accurate with natural frequency values giving errors below $10 \%$ for most cases. In addition to the dynamic analysis, for each composite action scenario, a static analysis with a unit load was conducted, in order to extract corresponding deflection measurements and to calculate the static-based LCA according to Eq. 2. More details on the numerical modelling can be found in [15].

\section{Results and Discussion}

To determine the loss of composite action, for the experimental and numerical timber composite beam model, first, $\alpha_{i j}$ values were calculated from the determined mode shape measurements by following the DI method and applying Eqs. 3 to 5. For the different composite action scenarios, various $\alpha_{i j}$ values were computed for each of the first five modes. The connection scenario with 33 screws was regarded as 'fully composite state' (no loss of screws/undamaged) and the 'partial composite states' with 17, 9, 5 and 3 screws were regarded as damaged states. Next, for each partial composite scenario, the dynamic-based LCAI was calculated following Eq. 6. Two sets of modes were considered for this calculation, i.e. modes 1 to 3 and modes 1 to 5 . This was done to study the effects of inclusion of mode shapes from higher modes, which is of special importance for experimental structures, where it is generally very difficult to determine accurate mode shapes of higher modes due to limitations in the number of measurement sensors. For this investigation, the mode shapes of modes 4 and 5 of the laboratory structure contained larger errors, which resulted in erroneous LCAI values, and hence, only results calculated from modes 1 to 3 are presented here. To provide a comparative measure for the composite action analysis, the static-based LCA, as defined in 
Eq. 2, was also calculated based on static analysis of the numerical beam models. The determined static-based LCA values for the investigated scenarios of 17, 9, 5 and 3 screws were calculated to be $1 \%, 2 \%, 7 \%$ and $25 \%$, respectively, based on a fully composite structure (100\%) with 33 screws.

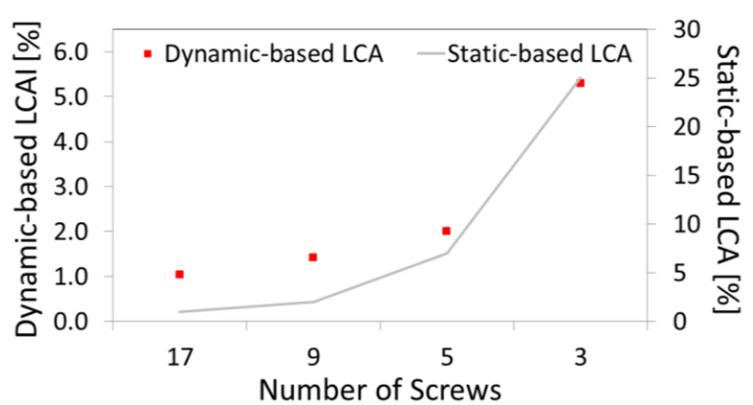

(a)

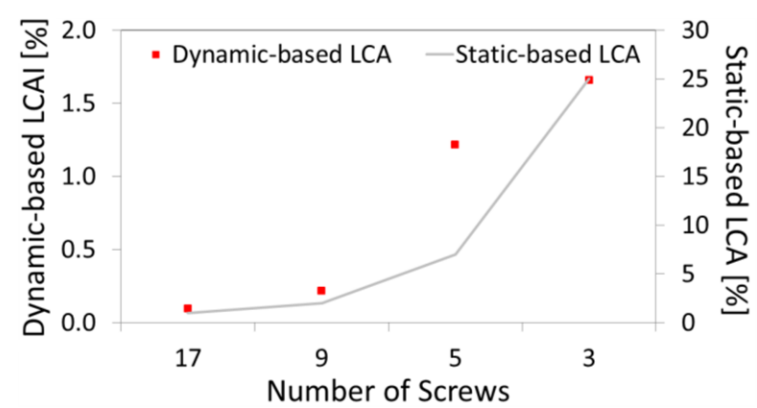

(b)

Fig. 3 Dynamic-based LCAI and static-based LCA of (a) laboratory and (b) numerical beam model.

The results of the dynamic-based LCAI plotted against the static-based LCA are shown in Fig. 3 for the four investigated partial composite states with 17, 9, 5 and 3 screws for (a) the experimental and (b) the numerical composite beam model. From the two figures, a clear correlation between the dynamic-based LCAI and the static-based LCA can be seen. Higher amplitudes of the dynamic-based LCAI clearly indicate a higher loss of composite action. For the experimental beam structure (Fig. 3 (a)), the dynamic-based LCAIs give some overestimations of the loss of composite action for the partial composite scenarios with 17, 9 and 5 screws. These overestimations are believed to be due to the experimental nature of the laboratory investigations, where the screw connections are not perfect fixtures as those simulated in the numerical model, and hence, the connection stiffness of the experimental structure will be lower than that of the corresponding numerical model. In this context it is noted that the static-based LCA, which is used as comparative measure, was only determined for the numerical model and not for the experimental structure, as the equipment for static load testing was not available during the course of the investigation. Nevertheless, it can be assumed that the testing results should be within acceptable range since a composite action of $100 \%$ for 33 screws is expected. For the results of the numerical beam structure (Fig. 3 (b)), the determined dynamic-based LCAI values are in very good agreement with the static-based LCA values for the composite scenarios with 17, 9 and 3 screws. The larger error for 5 screws is assumed to be due to the fact that the location of the two quarter-point screws is very close to the location of the node points of mode 3 . This causes a singularity issue in the ideal numerical model, which results in increased $\alpha_{i j}$ values for mode 3, and subsequently in an increase in the corresponding LCAI. Overall, the obtained results show a good correlation between the dynamically derived LCAI and the static-based LCA, and thereby demonstrate the effectiveness of the proposed composite action identification method.

\section{Summary}

This paper presented a new dynamic-based method for the identification of the loss of composite action of a composite flooring system due to the failure of shear connectors. The proposed approach is based on the Damage Index (DI) method and requires only mode shape measurements obtained from dynamic testing. The new algorithm is an extension of the DI method and is capable of assessing the overall condition of the connection system of a composite system. The proposed method was tested and validated on a numerical and experimental timber composite beam structure consisting of two timber components. To create different degrees of composite action, the composite beam was tested with different numbers of shear connectors to simulate the failure of connection screws. To evaluate the proposed new dynamic-based composite action method, the results obtained from the method were compared with outcomes from the traditional static-based load testing method. It was found that the results obtained from the new method were very promising and showed a clear correlation between the outcomes of the proposed dynamic-based method and that of the traditional static-based approach. 


\section{Acknowledgements}

The authors wish to thank the Centre for Built Infrastructure Research (CBIR), Faculty of Engineering, University of Technology, Sydney (UTS) for supporting this project. Within the Faculty of Engineering, the authors wish to express their gratitude to the staff of UTS Structures Laboratory for their assistance in conducting the experimental works. The Structural Timber Innovation Company (STIC) is gratefully acknowledged for their financial support.

\section{References}

[1] U. Kuhlmann, J. Schänzlin, A timber-concrete composite slab system for use in tall buildings, Structural Engineering International - Tall Timber Buildings, 18(2) (2007) 174-178.

[2] J. Pault, Composite action in glulam timber bridge systems, Master of Science Thesis, Colorado State University, Fort Collins, USA, 1977.

[3] R. Gutkowski, N. Miller, M. Fragiacomo, J. Balogh, Composite wood-concrete beams using utility poles: Time-dependent behaviour, Journal of Structural Engineering, 137(6) (2011) 625-634.

[4] S.W. Doebling, C.R. Farrar, M.B. Prime, D.W. Shevitz, Damage identification and health monitoring of structural and mechanical systems from changes in their vibration characteristics: a literature review, Los Alamos National Laboratory Report LA-13070-MS, Los Alamos, USA, 1996.

[5] H. Sohn, S.R. Farrar, F.N. Hemez, D. Shunk, D.W. Stinemates, B.R. Nadler, J.J. Czarnecki, A review of structural health monitoring literature 1996-2001, Los Alamos National Laboratory Report LA-13976-MS, Los Alamos, USA, 2004.

[6] E.P. Carden, P. Fanning, Vibration based condition monitoring: a review, Structural Health Monitoring, 3(4) (2004) 355-377.

[7] N. Stubbs, J.T. Kim, K. Topole, An efficient and robust algorithm for damage localization in offshore platforms, Proceedings of the 10th Structures Congress, Antonio, USA, 1992.

[8] F.C. Choi, J. Li, B. Samali, K. Crews, Application of the modified damage index method to timber beams, Engineering Structures, 30(4) (2008) 1124-1145.

[9] J.T. Kim, N. Stubbs, Improved damage identification method based on modal information. Journal of Sound and Vibration, 252(2) (2002) 223-238.

[10]N. Stubbs, J.T. Kim, C.R. Farrar, Field verification of a nondestructive damage localization and severity estimation algorithm, Proceedings of the 13th International Modal Analysis Conference, Nashville, USA, 1995.

[11]N. Stubbs, S. Park, Optimal sensor placement for mode shapes via Shannon's sampling theorem. Microcomputers in Civil Engineering, 11(6) (1996) 411-419.

[12]U. Dackermann, J. Li, B. Samali, Vibration-based damage identification in timber structures utilising the damage index method and neural network ensembles, Australian Journal of Structural Engineering, 9(3) (2009) 181-194.

[13]Z. Zabihi, B. Samali, R. Shrestha, C. Gerber, K. Crews, Serviceability and ultimate performance of long span timber floor modules, 12th WCTE Conference, Auckland, New Zealand, 2012.

[14]CADA-X, Modal analysis manual, LMS International, Leuven, Belgium, 1996.

[15]R. Rijal, Dynamic performance of timber and timber-concrete composite flooring systems, PhD Thesis, Faculty of Engineering and Information Technology, University of Technology, Sydney, Australia, 2013. 